\title{
Development of an anchorage prototype for CFRP stress-ribbon systems using 3D printing technique
}

\author{
Viktor Gribniak $^{1}$, Aleksandr K. Arnautov ${ }^{2}$, Arvydas Rimkus ${ }^{3}$ \\ ${ }^{1,3}$ Laboratory of Innovative Building Structures, Vilnius Gediminas Technical University, Vilnius, Lithuania \\ ${ }^{1,3}$ Department of Steel and Composite Structures, Vilnius Gediminas Technical University, Vilnius, Lithuania \\ ${ }^{2}$ Institute of Materials Mechanics, University of Latvia, Riga, Latvia \\ E-mail: ${ }^{3}$ arvydas.rimkus@vgtu.lt (correspondingauthor)
}

\begin{abstract}
Compared with other structure types, stress-ribbon systems are extremely simple though requiring massive anchorage blocks because of very large tensile stresses induced in the ribbons. Such structural systems are efficient in pedestrian bridges. A major drawback of these systems is related to corrosion of the steel ribbons. Unidirectional carbon fibre reinforced polymer (CFRP) has a high potential for replacing steel in the ribbons because of lightweight, high strength, and excellent resistance to corrosion and fatigue. Application of CFRP materials, however, faced serious problems due to construction of the anchorage joints. Thus, the anchorage system is the object of this research. Adhesive bonding is a simplest technology for joining structural components made of CFRP composites with polymer matrix. In the adhesion joints, the loads are transferred due to the shear effect. However, a relatively low inter-laminar shear strength of CFRP decreases effectiveness of the gripping systems. Brittle failure of the bond is often consequence of stress concentration. An innovative anchorage joint is proposed to control shear stresses by varying a local curvature of the contact surface. A natural shape of Nautilus shell was chosen for the gripping system, whereas a 3D printing technique was applied for the prototyping purpose. Mechanical behaviour of the anchorage prototypes made of printed polymeric material was investigated experimentally.
\end{abstract}

Keywords: high-performance materials, innovative bridge structures, non-metallic reinforcement.

\section{Introduction}

Stress-ribbon structural system is one of the oldest bridge types. It is efficient for pedestrian bridges (Juozapaitis et al., 2015). Compared with other structures, stress-ribbon systems are extremely simple - structures behaviour corresponds to a cable hanging freely between two supports; the gravity load stabilizes deformations. Such structural system requires massive anchorage blocks because of very large tensile stresses induced in the ribbons. A major drawback of these systems is related to corrosion of the steel ribbons. Excellent mechanical properties, high strength, and great resistance to corrosion and fatigue forced to consider unidirectional carbon fibre reinforced polymers (CFRP) as an alternative to steel in ribbons. Application of CFRP materials, however, faced serious problems due to construction of the anchorage joints. The anchorage system of fibre reinforced carbon polymer (CFRP) ribbons is the object of this research.

The experimental results of the tensile load transmission from the gripping devices to the CFRP strip are presented in this study. Such devices are necessary for pre- and posttensioning of CFRP strips. A similar gripping system can be used for tensile tests of CFRP since the idea of the developed gripping system is related to control of the shear stresses acting at the contact surface varying curvature over the gripping surface. Allowable curvature of the contact surface is closely related to flexural stiffness of the gripped strip because of formation of additional stress due to the bending effect. The additional stress component increases with increasing flexural stiffness of the anchorage strip that requires a reduction of the maximum allowable curvature of the contact surface and increases dimensions of the proposed gripping system making it inapplicable for practical purposes.

The load transmission is realized by the use of gripping/anchorage systems. The tensile load is transferred to a tensioned strip by the shear stresses operating on its surface because of micro slip-induced friction of the strip in the gripping device. The actual ratios between the surface and cross-sectional areas of the strip seems to be very appropriate for friction anchors, where the strip is clamped between wedges or bolted plates (Portnov, Kulakov, \& Arnautov, 2006; Mohee, Al-Mayah, \& Plumtree, 2016). In both cases, distribution of the shear stresses over the gripping surface is not

(C) 2019 Authors. Published by VGTU Press. This is an open-access article distributed under the terms of the Creative Commons Attribution (http://creativecommons.org/licenses/by/4.0/) License, which permits unrestricted use, distribution, and reproduction in any medium, provided the original author and source are credited. 
uniform: a stress peak is appearing near the entry into the anchor or gripping device. This peak creates a concentration of normal longitudinal stresses in the near-surface layers of the strip, which considerably exceed the mean stress in the tensioned strip. This leads to a premature failure of the tensioned strip near the entry into the anchor and does not allow one to achieve the real maximum load-carrying capacity corresponding to the attainment of the tensile strength by stresses uniformly distributed on the working cross-sectional area of the strip. However, the existing gripping techniques can solve this problem only partially (Kulakov, Tarnopol'skii, Arnautov, \& Rytter, 2004; Burtscher, 2008). The anchorage systems enable a certain decrease in the concentration of shear stresses, but the stress concentration is not disappearing completely. Distribution shape of the shear stresses along the tensioned strip, in essence, does not change, i.e. the maximum stress is localized at the entry into the gripping device. The surface stresses decrease moving inside the device. Application of adhesive or compliant interlayers in gripping devices practically excluded the possibility of repeated use of the anchor parts being in an immediate contact with the surface of a tensioned element. Therefore, there is a need to continue the development of sufficiently simple and effective gripping devices for tensioning different kinds of flexible composite strips.

This paper describes a design concept of gripping device for tensioning a flexible composite strip. The grips transfer tension mainly by tension-induced frictional forces. To control shear stresses in composite strip, an innovative anchorage joint with a shape of Nautilus shell (Figure 1) is proposed. A 3D printing technique was implemented for the production of the gripping system prototype. Mechanical behaviour of the anchorage prototype made of printed polymeric material was determined experimentally.
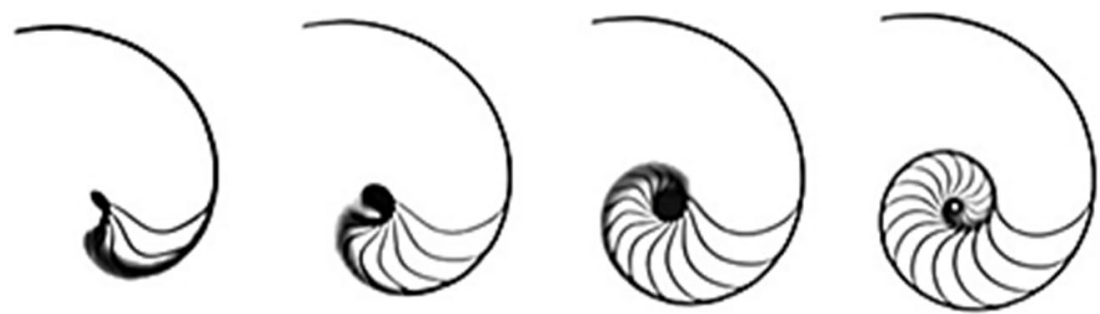

Figure 1. The Nautilus shell grow

\section{Theoretical background}

A two-stage loading procedure is considered. At the first step, the flexible strip is placed on a curved surface of the grips. The inner end of the strip is fastened; a small external load is applied ensuring contact with the gripping system. At the second step, an external tension load is applied to the free end of the CFRP strip. The strip transfers tension to the grips because of frictional forces. Special grips with a varying curvature were developed to transmit the tensile load to the strip by gradually increasing shear stresses (without peaks). The developed gripping device for a flexible composite strip is shown in Figures 1 and 2.

A natural shape of Nautilus shell (Figure 1) was chosen to control shear stresses because of steady decrease of curvature of the contact surface and consequential increase of the bond strength. The following equation describes a spiral curve in polar coordinate system (Shevelev, Marutaev, \& Shmelev, 1990):

$$
r=\sqrt{\Phi} \cdot \exp \left(\frac{2 \varphi}{\pi} \ln \sqrt{\Phi}\right) ; \quad \Phi=\frac{1+\sqrt{5}}{2}
$$

where $r$ and $\varphi$ determine the polar coordinates (radius and rotation angle); $\Phi$ is the Golden Ratio (also known as the Golden Section, Golden Mean, Divine Proportion). The initial radius value $r_{0}$ depends on material properties (flexural stiffness and strength of the strip material). In the considered case, a low resistance of the CFRP sheet determines the minimum radius $r_{0}$ that was set $60 \mathrm{~mm}$ to avoid stress localization in the CFRP grip due to local flexure (owning the actual flexural stiffness of the $0.5 \mathrm{~mm}$ thickness CFRP strip). The minimum radius was selected to limit the total stresses due to the combined action of the tension and bending effects in the strip. The total stress should not exceed the local strength of the CFRP strip. It is also necessary to take into account the reduction of the tensile stress in this area due to friction.

Following equations represent the angle of the initial point of the spiral grip:

$$
\varphi_{0}=\frac{\pi}{2} \cdot\left(\frac{\ln r_{0}}{\ln \sqrt{\Phi}}-1\right)
$$

The Cartesian coordinates and the polar coordinate system are related through the transformation:

$$
\left\{\begin{array}{l}
x=r \cos \varphi \\
y=r \sin \varphi
\end{array} .\right.
$$


A gripping system having a spiral shape of the contact (frictional) surface adapted to accommodate the flexible strip. In longitudinal section of the gripping device, the curvature of the contact surface gradually decreases from the internal grips and disappears at the entry of the anchorage device. In such a gripping system, shear stresses at the strip surface vary smoothly along the contact surface. The varying curvature of the surface permits loading of the strip by frictional shear and transverse normal stresses present on its surfaces, which are induced by tensioning the strip. The absence of stress peaks allows increasing the load transmitted to the strip.

Transmission of the tensile load to the flexible strip by tensioning the strip inside the gripping system allows to prevent stress concentration on the surface of the strip at the entry point. In such a way, the stress peaks, which usually lead to premature rupture of the strip in the traditional grips, are excluded. The load transmitted to the strip using suggested gripping device may be increased considerably. The gripping device allows to avoid peaks of shear and normal stresses on strip surface, as well as the concentration of longitudinal tensile stresses in the near-surface layers of the strip. This paper presents an idea of the design of a gripping device for tensioning flat composite strips. The grips transfer tension mainly by tension-induced frictional forces, just as threads bent over a curved surface. The friction on thread surface is controlled by changing the curvature of the contact surface. Figure 2 a shows a scheme of the developed grip layout. The red line indicates the CFRP strip. An internal conical supplemental grip is placed inside the internal rectangular opening as shown in Figure $2 \mathrm{~b}$. These grips were used to fix the CFRP strip at the preloading stage. The supplemental grips were also used to prevent a sudden failure of the gripping system due to lack of the adhesive contact. Deformations of the CFRP strip were monitored in the gap between the internal edge of the spiral surface and the supplemental grips.

Common steel gips have a low friction/cohesion coefficient to CFRP materials. Therefore, a 3D printed thermoplastic polymeric material was used to manufacture the grips. A low deformation modulus of the polymeric material not only increases the cohesion, it also reduces local stress concentrations due to high deformability of the material in orthonormal direction (to the bonding surface). Shkundalova, Rimkus, and Gribniak (2018) investigated the mechanical properties and tensile failure of four thermoplastic polymeric materials: acrylonitrile butadiene styrene (ABS), polylactic acid (PLA), high impact polystyrene (HIPS), and polyethylene terephthalate (PETG). The investigation shown, that the ultimate strain of the specimens made of ABS, HIPS and PETG were localized between printed filaments causing local brittle failure, while PLA experienced fundamentally different ductile failure. Based on these results and the fact that PLA provides relatively high nominal tensile strength $(28.1 \mathrm{MPa})$, body of the developed gripping system were printed from PLA material.

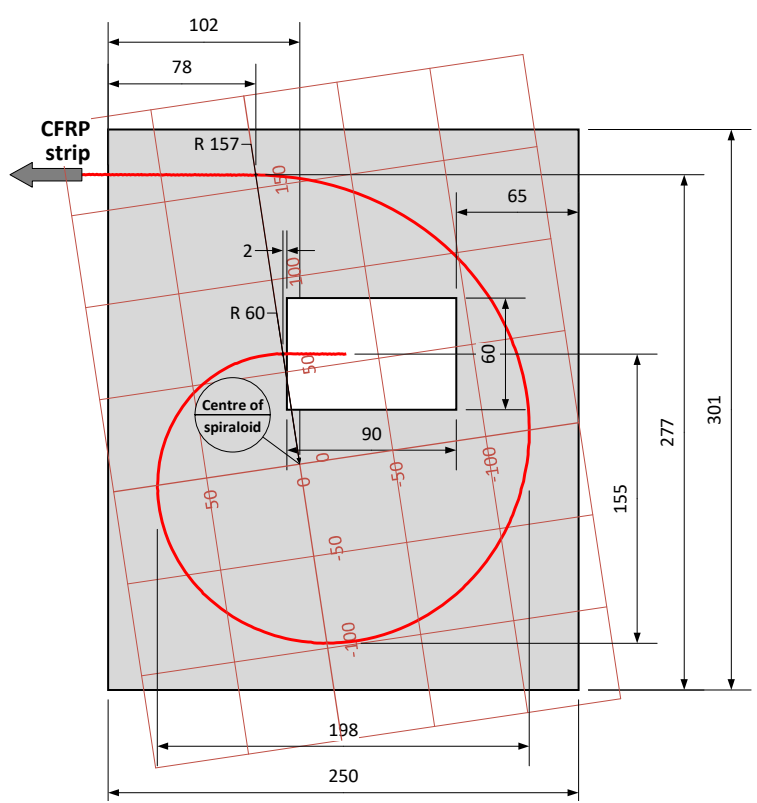

a)

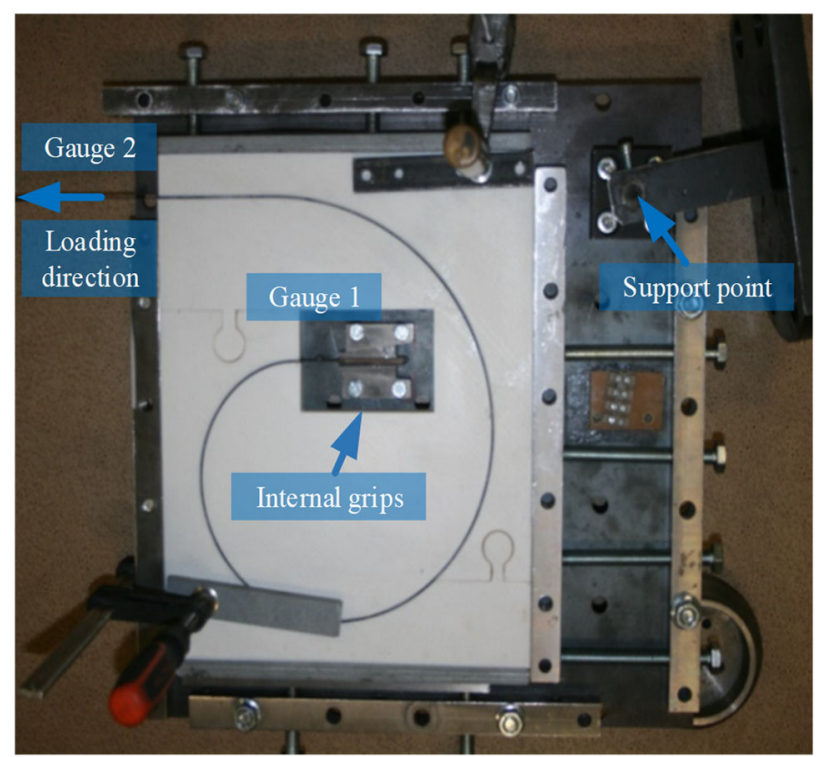

b)

Figure 2. Layout of the spiral shape gripping system: a) a scheme (dimensions are in $\mathrm{mm}$ ); b) loading apparatus

\section{Tensile tests and discussion}

This paper describes an attempt to design an anchorage device for tensioning a composite strip preventing concentration of shear stresses at the gripping surface. The gripping system was developed for a prefabricated $10 \mathrm{~mm}$ wide and $0.5 \mathrm{~mm}$ thickness carbon fibre-reinforced polymer (CFRP) strip. Nominal density of the strip $=1.3 \ldots 1.5 \mathrm{~g} / \mathrm{cm}^{3}$. The CFRP strips were not tested assuming mechanical and physical properties specified by the producers: tensile strength $=$ 
$400 \ldots 500 \mathrm{MPa}$, compressive strength $=200 \ldots 320 \mathrm{MPa}$, modulus of elasticity $=28 \ldots 40 \mathrm{GPa}$, Barcol hardness $=$ 25...45. To implement the suggested idea, the gripping device shown in Figure 2 a was designed. The profiled parts of the device were fabricated from a polymeric material (PLA) plate of thickness $15 \mathrm{~mm}$ (Figure 2b). Eq. (1) described the spiral shape of the profile.

CFRP specimens of $1000 \mathrm{~mm}$ length made by pultrusion from a high-strength unidirectional CFRP/epoxy composite were prepared for the tests. To prevent the CFRP strip from failure inside the self-tightening wedge grips, $1.5 \mathrm{~mm}$ thick and $45 \mathrm{~mm}$ length GFRP tabs were glued on both ends of the strip specimens by a two-component polyurethane structural adhesive. The value of tensile strains across the strip length was measured by strain gauges, glued between wedge and profiled grips and outside of the profiled grip. Preparing for tensile tests, a CFRP strip specimen with glued strain gauges was inserted between the profiled grips. In order to avoid separation of the grips during tensioning of the strip, the grips were closed by applying a slight compression by means of adjustment screws (see Figure $2 \mathrm{~b}$ ). To create a uniform compressive force during closing the profiled grips, the adjustment screws were tightened with a torque wrench creating a torque of $12 \mathrm{Nm}$. After placing the strip in the gripping devices, the ends of the strip were fixed in self-tightening wedge grips.

As shown in Figure 2b, the plastic body of the spiral grips (grey material) was fragmented. The body was printed in three parts due to limitations of the 3D printer. Identical printing manner was applied for each part: two solid "shells" were printed on the perimeter of the specimen. The inner part of the sample was printed with $60 \%$ infill at rectilinear raster orientation (such printing layout is commonly used for prototyping purposes). It is important to note, that first and last two layers of the specimens were printed with $45^{\circ}$ angle oriented $100 \%$ infill. The mechanical properties of the printed material were determined experimentally by performing tensile test of typical dumbbell-shaped samples. Shkundalova et al. (2018) reported the geometry of the samples, printing and testing parameters. Tensile strength of the printed PLA material was equal to $37.7 \mathrm{MPa}$, while modulus of elasticity $=5.8 \mathrm{GPa}$. The parts of the spiral grips as well as the dumbbell-shaped samples were printed using Prusa $i 3 M K 3$ printer with identical printing parameters: extrusion nozzle temperature $=215^{\circ} \mathrm{C}$; printing bed temperature $=60{ }^{\circ} \mathrm{C}$; print speed $=40 \mathrm{~mm} / \mathrm{s}$; infill density $=60 \%$.

The gripping devices with the inserted strip specimen was mounted vertically in a servo-hydraulic testing machine MTS 809.40, where the specimen was centred and fixed by means of special discs. The crosshead speed of the testing machine was $2 \mathrm{~mm} / \mathrm{min}$ and ensured loading of the specimen at the initial stage without jerks. During the test, the relationships between the applied load and strip strains were monitored. The first attempt to perform a test was unsuccessful. The unidirectional CFRP strip was pulled out of the wedge grip. Measurements showed that the coefficient of friction between the contact surfaces of polymer material and the unidirectional CFRP/epoxy strip was unexpectedly small.

An effective way to increase the strength of joints without damaging CFRP members is increasing the friction between CFRP contact surfaces (Katsumata, Mizutani, Todoroki, \& Matsuzaki, 2010). Sandpaper has a high friction coefficient independent of the normal stresses acting on the contact area, unlike the coefficient of other high-friction materials currently available for joints, such as rubber gaskets and adhesive tapes. The measured values of the coefficients of friction for these configurations were as follows (Katsumata et al., 2010): untreated surface, $f_{r}=0.36$; sandblasting $(\# 100)=0.42$; sandblasting $(\# 800)=0.39$ with a sandpaper gasket $(\# 40)=0.66$; with a sandpaper gasket $(\# 240)=0.68$. The presence of sandpaper is the most effective method for increasing the coefficient of friction. To increase the coefficient of friction, sandpaper was glued on the contact surface of the profiled grips. This ensured the failure of the CFRP strip in the gauge section.

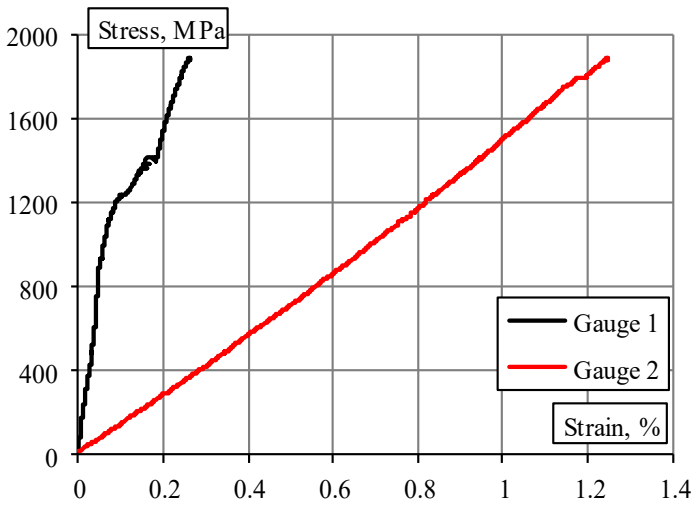

a)

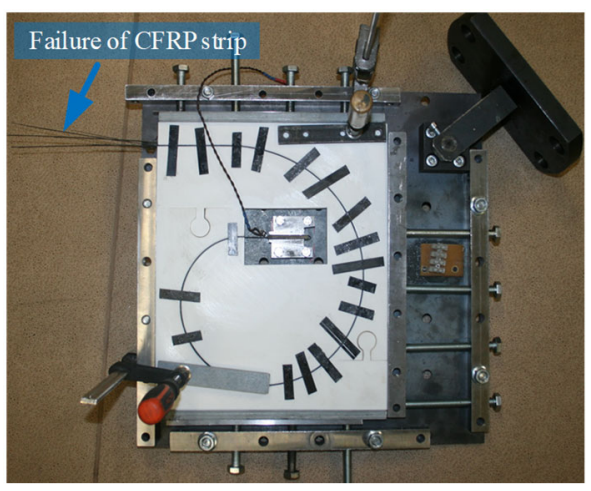

b)

Figure 3. Results of tensile test of flexible CFRP strip: a) stress-strain diagrams of internal (Gauge 1) and external (Gauge 2) strain-gauges; b) failure of the strip 
The stress-strain relationships of CFRP strip are shown in Figure 3a. The diagram demonstrates substantial differences between deformations monitored inside the spiral grip (Gauge 1) and outside the grip (Gauge 2). This difference is a consequence of friction between the contact surfaces of polymer material and CFRP strip. The maximum tensile stress in the gauge section of the tested strip in the gripping device with an enhanced friction coefficient was 1892.9 MPa, modulus of elasticity $=144.2 \pm 1.8 \mathrm{GPa}$, ultimate strain $=1.25 \pm 0.04 \%$ (the average value from two tests). Figure $3 \mathrm{~b}$ shows the failure of CFRP stip. It was localized outside of the gripping system that proves principles used for design and manufacturing of the spiral grip.

To verify the test results obtained using the designed gripping device, three flat specimens made of the same unidirectional CFRP strip were tested in uniaxial tension according to the ASTM D 3039/ D 3039 M-07 standard. The following values of mechanical characteristics were found: modulus of elasticity $=138.2 \pm 0.8 \mathrm{GPa}$, tensile strength $=$ $1897.4 \pm 49 \mathrm{MPa}$, and the ultimate strain $=1.27 \pm 0.04 \%$. Obtained results practically coincide.

\section{Conclusions}

This work is dedicated for development of design concept of a gripping device for tensioning a flexible composite strip. The tensile load is transferred to the gripping device because of friction. A variable curvature of the contact surface was used to avoid concentration of the shear stresses. The design model is based on the simplified assumptions of a constant coefficient of friction between the contact surfaces of the strip and grips and perfectly elastic behaviours of the grips. An innovative anchorage joint with a shape of Nautilus shell was proposed. A 3D printing technique was implemented to manufacture the gripping system prototype. Mechanical behaviour of the anchorage prototype was determined experimentally. The test results demonstrated ability of the proposed gripping system to reduce the stress concentration: failure of the CFRP strip was localized outside the gripping system.

The proposed method of transmission of a tensile load on a tensile strip is suitable for design of anchors for prestressed strips as well as for gripping systems for mechanical testing of flexural strips. The stress-strain behaviour of the gripping system is closely related with flexural stiffness and strength of the strip, and cohesion parameters of the contact surfaces. Therefore, adequate design of the surface shape must take into account actual properties of the materials. A friction behaviour of high-strength composite strips (especially CFRP) is necessary for further development of the proposed design method.

\section{Acknowledgements}

The authors gratefully acknowledge the financial support provided by the Research Council of Lithuania [project number S-MIP-17-62].

\section{References}

Burtscher, S. L. (2008). Wedge anchorage for CFRP strips. Journal of Composites for Construction, 12(4), $446-453$. https://doi.org/10.1061/(ASCE)1090-0268(2008)12:4(446)

Juozapaitis, A., Merkevičius, T., Daniūnas, A., Kliukas, R., Sandovič, G., \& Lukoševičienè, O. (2015). Analysis of innovative twospan suspension bridges. The Baltic Journal of Road and Bridge Engineering, 10(3), 269-275. https://doi.org/10.3846/bjrbe.2015.34

Katsumata, T., Mizutani, Y., Todoroki, A., \& Matsuzaki, R. (2010). A fundamental study on static strength improvement of CFRP bolted joints by increasing friction force. Journal of Solid Mechanics and Materials Engineering, 4(6), 711-719. https://doi.org/10.1299/jmmp.4.610

Kulakov, V. L., Tarnopol'skii, Y. M., Arnautov, A. K., \& Rytter, J. (2004). Stress-strain state in the zone of load transfer in a composite specimen under uniaxial tension. Mechanics of Composite Materials, 40(2), 91-100. https://doi.org/10.1023/B:MOCM.0000025483.37317.e2

Mohee, F. M., Al-Mayah, A., \& Plumtree, A. (2016). Anchors for CFRP plates: state-of-the-art review and future potential. Composites Part B: Engineering, 90, 432-442. https://doi.org/10.1016/j.compositesb.2016.01.011

Portnov, G. G., Kulakov, V. L., \& Arnautov, A. K. (2006). A refined stress-strain analysis in the load transfer zone of flat specimens of high-strength unidirectional composites in uniaxial tension 1. Theoretical analysis. Mechanics of Composite Materials, 42(6), 547-554. https://doi.org/10.1007/s11029-006-0065-9

Shevelev, I. Sh., Marutaev, M. A., \& Shmelev, I. A. (1990). Section divine, three views on the nature of harmony. Moscow: Stroizdat (in Russian).

Shkundalova, O., Rimkus, A., \& Gribniak, V. (2018). Structural application of 3D printing technologies: mechanical properties of printed polymeric materials. Science - Future of Lithuania, 10, 1-8. https://doi.org/10.3846/mla.2018.6250 